\title{
Solution to Cleopatra's Cocktail challenge
}

\author{
Prudence J. Jones
}

Published online: 30 December 2010

(C) Springer-Verlag 2010

The winner of the Cleopatra's Cocktail challenge (published in issue 398/5) is:

D. Fatta-Kassinos, Department of Civil and Environmental Engineering, School of Engineering, University of Cyprus, 1678 Nicosia, Cyprus.

The award entitles the winner to select a Springer book of his choice up to a value of $€ 75$.

Our Congratulations!

\section{Solution}

Pearls are made up of calcium carbonate therefore the reaction between a pearl and vinegar is an acid-base reaction and vinegar would, as Pliny describes, destroy a pearl. The calcium carbonate in a pearl reacts with the acetic acid (vinegar) to produce calcium acetate, water, and carbon dioxide:

$\mathrm{CaCO}_{3}+2 \mathrm{CH}_{3} \mathrm{COOH} \rightarrow \mathrm{Ca}^{2+}+2 \mathrm{CH}_{3} \mathrm{COO}^{-}+\mathrm{H}_{2} \mathrm{O}+\mathrm{CO}_{2}$.

If the precise amount of acetic acid needed to react with the calcium carbonate is used, all of the acid is neutralized and the cocktail would be quite palatable (the pearl lends a somewhat fishy taste). The reaction occurs very quickly with powdered calcium carbonate. In a pearl, however, the calcium carbonate is deposited between layers of an organic matrix, which reduces the rate of reaction. Experiments reveal that crushing

This article is the solution to the Analytical Challenge to be found at http://dx.doi.org/10.1007/s00216-010-4149-y.

\section{P. J. Jones $(\triangle)$}

Department of Classics and General Humanities,

Montclair State University,

Montclair, NJ 07043, USA

e-mail: jonespr@mail.montclair.edu the pearl increases the reaction rate, as does using an excess of vinegar and increasing the temperature at which the reaction is carried out. Interestingly, increasing the concentration of acetic acid beyond that found in vinegar fermented from wine (ca. 10\%), reduces the rate of the reaction.

A pearl weighing approximately $1 \mathrm{~g}$ takes 24 to $36 \mathrm{~h}$ to fully react with a $5 \%$ solution of acetic acid, a concentration identical to that of white vinegar sold in supermarkets. ${ }^{1}$ After the pearl has reacted fully with the vinegar, a small amount of translucent, gel-like material remains. This organic material represents the protein matrix of the pearl and does not react with the acid, but is softened by it.

Concentrations of acetic acid higher than those that can be obtained by fermenting wine hinder the reaction. In experiments using concentrations of acetic acid higher than those found in vinegar, experiments show that the reaction rate decreases. In a $5 \%$ acetic acid solution, powdered calcium carbonate took 3 min to react fully ${ }^{2}$; in a $25 \%$ solution, the reaction took $35 \mathrm{~min} ; 100 \%$ acetic acid failed to react with powdered calcium carbonate within $24 \mathrm{~h}$. In $100 \%$ acetic acid, a pearl's outermost layer of organic material is softened within $24 \mathrm{~h}$, but the disintegration does not proceed beyond that point and there are no bubbles of carbon dioxide to indicate that a reaction is occurring. These further experiments indicate that not only are the concentrations of acetic acid found in vinegar adequate to react with a pearl, they are ideal.

\footnotetext{
${ }^{1}$ In all trials, a volume of acetic acid solution in excess of the stoichiometric amount determined by the mass of the calcium carbonate was used. Using an excess of acid ensures that any lack of reaction is not due to the quantity of reagents present.

${ }^{2}$ Powdered calcium carbonate reacts more readily than a pearl with acetic acid due to the calcium carbonate powder's greater surface area and lack of organic material.
} 